Trieste

\title{
VEDICA MINORA
}

Nell' atto dell' erezione del palo centrale della capanna sacrificale vedica (W.Caland-v.Henry, L'Agniștoma, Paris 1906-1907, p.94 e segg). viene recitato un versetto (TS $1,3,1$, i) che dice

dyutānás tvā mārutó minotu mitrắvárunayor dhruvéna dhármanāa

ossia "Che Dyutāna Māruta (il figlio o discendente dei Maruti) ti innalzi secondo il dharma fissato di Mitra-e-Varuna"; cfr. TS 6,2,10,4. Il versetto, con irrilevanti differenze testuali, è proprio anche della Vảjasaneyi Samhitã $5,27 \mathrm{~b}$. L' accordo tra le due tradizioni yajurvediche è peraltro rotto da MS $1,2,11$ che recita

nitānás tvāa mārutó ni hantu mitrấvárunau dhruvéna dhármanāā

"N.M. ti possa inserire (scil.: nel buco preparato nel terreno), oh Mitrae-Varuna, con fissato dharma!"; KS 2,12 presenta il piu corretto gen. duale dei teonimi ("... secondo il dharma di M.V."), laddove 'più corretto' equivale, per ora, $a$ ' più comprensibile'.

Non suscita eccessive preoccupazioni il fatto che l'attenzione liturgica si fissi su aspetti diversi del medesimo momento rituale, e che di conseguenza sia leggermente divergente lo yajus, quanto piuttosto la differenza del nome proprio, soprattutto se si considera che il versetto proviene dalle parti sicuramente piu antiche di MS e $\underline{K S}$ (II libro = madhyama!), dunque una lectio di buona credibilità e che potrebbe al limite configurarsi come difficilior. Anche senza riprendere il difficile problema dei rapporti TS/

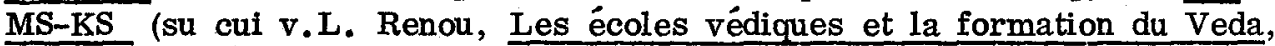
Paris 1947, p.129 e segg.; J. Gonda, Vedic Literature, Wiesbaden 1975, p. 323 e seg.; ivi ricca bibl.), è chiaro che il problema sembra avere una sua certa consistenza filologica; ma non basta, perché evidentemente la questione è correlata alla qualificazione della figura di Dyutāna Māruta - peraltro estremamente scialba - nella mitologia vedica.

Le testimonianze relative a D.M. sono state raccolte da A.A. Macdonell A.B. Keith, Vedic Index of Names and Subjects, London 1912, I, p. 382, e stando ai loro dati ed ai testi che essi citano pare chiaro che già gli antichi avevano idee estremamente incerte, se non addirittura inconsistenti, su tale personaggio. Nello $\underline{\text { SB }} 3,6,1,16$ (commento al passo di VS sopra ricordato) D. M. è identificato con il vento, mentre 1 ' anukramani $\overline{\text { lo }}$ 
considera dubitativamente un rşi, forse autore di RV 8.96. PB 17,1,6 sembrerebbe considerario autore di un canto liturgico (săman) di notevole importanza nel vrātyastoma e gli attribuisce il titolo di grhapati del vrātya $(17,1,7)$. Di quest'ultimo fatto si era occupato diffusamente J.W. Hauer, Der Vrätya, Stuttgart 1927, passim, all'interno della sua tesi secondo la quale i vräya erano degli estatici, predecessori degli yogin contemporanei; la tesi è inaccettabile, e y. l'intelligente messa a punto di A.C.Banerjea, Studies in the Brăhmanas, Delhi 1963, p.81 e segg. (v. anche le mie "Ricerche di antichita indeuropee", Trieste 1979, p.57). Comunque sia, neppure al Hauer era riuscito in alcun modo di dare consistenza alla figura di D.M., laddove, alla luce di quanto è stato ottimamente dimostrato da J.C. Heestermann "IJ" 6 (1962) pp.1-37 circa gli strettlssimi legami intercorrenti tra compagine mitica del Maruti e fenomeno socio-culturale vrātya, è perfettamente lecito sospettare che Dyutāna sia stato inserito in ambiente vrātya proprio in virtù del suo patronimico (Māruta), con una sorta di sillogismo teologizzante. In altre parole parrebbe proprio che D.M. come figura mitica sia evanescente, laddove sia ben reale come lectio filologica. Cio pud far sorgere il sospetto che il versetto sia testualmente corrotto e che la corruzione risalga ad epoca molto antica.

Nitāná non ha sèguito come nome mitico, ma non è vox nullius in quanto compare in AV 6,139,1 col senso di "shoot of a plant which grow downwards" (Monier-Williams; v. anche J. Gonda, The vedic God Mitra, Leiden : 1972, p. 66): e qui non si possono omettere importanti considerazioni.

Il pilastro del sadas è di udumbara (Ficus glomerata) e lo stesso vale per l'importantissimo pilastro sacrificale (TS $2,1,1,6$ ), ma la descrizione fornita da nitäná sembrerebbe piuttosto confarsi al nyagrodha (Ficus indica); in realta è perfettamente chiaro che il riferimento è di ordine prettamente mistico-cosmologico, in quanto il palo è immagine dell'Albero Cosmico = axis mundi, Su questo tema ha scritto pagine molto belle H. Lommel, "Paideuma" 6 (1958) pp.490-499: tra $i$ tanti passi che si possono invocare a sostegno dell'identificazione basti TS $1,3,5$, g: "Con la (tua) / cima non graffiare il cielo, con il mezzo non arrecar danno all'atmosfera, sii unito alla terra!", così si dice all'albero che sta per essere abbattuto ed il cui legno diventerà il palo. La presenza dell'Albero Cosmico sul luogo del sacrificio è il segno che in tale area si verifica una 'rottura di livello cosmologico', ossia i piani cosmici (cielo/terra/aldilà) si fondono in uno solo (cfr. M. Eliade, Traité d'histoire des religions, Paris 1948, cap. X; id.; Le mythe de 1'éternel retour, Paris $1969^{2}$, cap. I). Anche 1'altare è considerato nel Rigveda il 'centro del mondo', cosi come centrale è l'oscura e tenebrosa dimora del dio Varuna, nella quale egli sostiene il pilastro cosmico (v.le mie "Ricerche..", cit., p.135 e segg e p. 150-151). Come ultima, e più puntale, giustificazione diremo che tale Albero Cosmico è pensato in India rivol to verso il basso, in posizione rovesciata ( $P$. Thieme, 
Untersuchungen zur Wortkunde und Auslegung des Rigveda, Halle 1949, p. 67 e segg.; F.B.J. Kuiper, "IIJ" 8(1964) p.116 e segg.). La presenza di nitāná è dunque perfettamente motivata nel versetto yajurvedico che cl occupa: ma c'è di più. A. Hillebrandt, Vedische Mythologie, Breslau 1929², II, pp. 279-280, ha espresso la fondata opinione che dev'essere esistita anticamente una leggenda (a noi non esplicitamente documentata) che legava l'aśvattha ai Maruti; come è noto, taluni avevano ritenuto che tale collegamento, alquanto frequente nella letteratura liturgica, fosse derivato da un'esegesi errata di $\mathrm{RV} 1,135,8$. A mio avviso l'osservazione del Hillebrandt è validissima, ma comunque sia dobbiamo riconoscere che se travisamento c'è stato (e a partire dal passo rigvedico), ciò dev' essere avvenuto prima della sistemazione definitiva del testo, come noi lo conosciamo, della TS: ivi infatti troviamo l'inciso $(3,5,7,2)$ märutò 'śvatthó "l'a. marutiano". Con ciò si giustifica sia concettualmente sia morfologicamente il sintagma nitānás... mārutó del nostro versetto, poiché a questo punto è chiaro che nulla ci costringe a considerare il secondo termine come patronimico.

Credo di poter proporre una restituzione accettabile del versetto sulla base di quanto sin qui è stato detto; il punto centrale è l'accusativo tvăa, che a mio avviso e errore (agevolmente giustificabile) della tradizione orale e della recitazione per il nominativo tvám, ossia nitānás * tvám mārutó; abbiamo cioè una frase nominale di ottima credibilità, "Tu sei il pollone all'ingiù marutiano": dopo di essa il senso esige una pausa e quanto segue ("che egli ti innalzi ...") dev'essere inteso come un invito augurale rivolto all' officiante che sollevava il palo. La nostra restituzione ha il vantaggio di spiegaanche un altro fatto, ossia la personificazione (arbitraria, e di ciò appresso) di Dyutāna Māruta poiché nel momento stesso in cui /tvám-mārutó/viene sentito o detto / tvăm-mo/ o/tvā- $\mathrm{m}$ \% è necessario cercare un soggetto per la forma verbale che segue ed il riconoscimento di esso in una sequenza di nominativi - dei quali uno interpretabile come patronimico! - è del tutto spontaneo.

Dyutāná - sembra di trasparente lettura morfo-etimologica, ossia un part. aor.rad.medio della radice dyut - "brillare", spesso usato come aggettivo, e non escludo del tutto che questo possa essere il senso dell'appellativo nel nostro caso, ma la cosa mi pare invero ben poco soddisfacente, per cui due mi sembrano le possibili soluzioni - sempreché, beninteso non si voglia considerarlo una crux: o d. è una refezione (pseudo) esplicativa del raro nitāná (ma non comprendo i modi di tale paretimologia, perché l'assonanza è meno che parziale), oppure abbiamo a che fare con un composto $*$ dyutāná, formato con un elemento * tāná- "pollone", garantitoci da ni-tāná, ma non altrimenti attestato. Il significato di siffatto composto ("pollone del 
cielo") sarebbe oltremodo consolante, considerate le implicazioni mitologiche sopra esposte. 1

Mi si permetta qualche cursoria osservazione sull'aspetto rituale del pilastro (/axis mundi) nell'India vedica, senza alcuna aspirazione alla completezza. Lo yūpa ha una funzione precisa nel sacrificio animale (J.Schwab, Das altindische Thieropfer mit Benützung handschriftlicher Quellen dargestellt, Erlangen 1886), poiché esso è il punto in cui si concentrano tutte le forze religiose che sono in gioco nel sacrificio (v.il classico lavoro di $\mathrm{H}$. Hubert e M. Mauss in "Année sociologique" 2,1899, p.29 e segg.). Non si pud certo dire lo stesso del palo centrale del sadas sopra ricordato, che pure riceve tante cure liturgiche (tra l'altro con l'utilizzo di mantra identici a quelli recitati per l'erezione dello yūpa sacrificale!) e che sarà trascurato per tutto il resto del rito: non basta come spiegazione il fatto che esso sia figura dell'axis mundi. Si può avanzare il fondato sospetto che nel secondo caso da noi ricordato si sia di fronte ad una funzione scaduta, ossia in epoche anteriori (e forse non di poco) alla fissazione del rituale śrauta esistevano riti di erezione del palo=Albero Cosmico poi perduti o travisati. Così, ad esempio, nella cerimonia del rājasūya ( J. C. Heesterman, The ancient Indian royal consecration, The Hague 1957, p.96 e segg.) l'innalzamento dell'axis mundi è nettamente evocato, pur senza la presenza effettiva di un pálo, attraverso l'alzarsi e l'abbassarsi delle mani del sacrificante ${ }^{2)}$. Ma il caso più clamoroso è offerto dal vājapeya,

1 Ricordo che in VS $6,6 \mathrm{~b}$ si dice ad un frammento dello yūpa "Tu sei il fịglio del cielo. "(diváh sunúr asi). Per altri esempi di false personificazioni (dovute ad errori esegetici o sim.).v.J.Gonda, Vedic Literature, Wiesbaden 1975, p.76.

2 Credo che tale sia anche il senso dell" "alzare le mani" del dio Savitar, un gesto talmente celebre che talora viene attribuito ad altri del con esplicito richiamo a Savitar (es RV 1,95,7, rivolto ad Agni). L'alzarsi delle mani (ed il loro abbassarsi) può essere correlato anche al ciclo quotidiano del sole; con cio non voglio affatto porre in indebita evidenza il carattere solare del dio (un carattere peraltro indubitabile), poiché Savitar si appalesa in primo luogo come divinità stimolatrice del movimento (sulle connessioni ideologiche vediche tra movimento e vita v.M.Durante, Sulla preistoria della tradizione poetica greca, II, Roma 1976, p. 143 e R.Lazzeroni, "SSL" 15,1975, p. 1 e segg.), movimento fisico (muove/fa riposare gli uomini e la na- 
nel quale il palo sacrificale serve per riti di ascensione, debitamente mimati dal sacrificante e da sua moglie, nei quali vengono recitati mantra come TS $1,7,9, \mathrm{e}$, súvar devấ agamnā "abbiamo raggiunto il cielo luminoso, gli dei": è mia opinione (sulla quale tornerò altrove) che siffatta concezione del palo (/Albero Cosmico/axis mundi) come mezzo di comunicazione tra mondo terrestre e mondo degli dei (le connessioni funerarie sono tuttavia ben presenti!) sia ben antica tra $i$ popoli vedici e sia di ascendenza sciamanica, fatto questo che non vediamo più chiaramente riflesso nella facies religiosa delle Samhitā è che dunque e molto antico.

Poiché il palo è il segno cosmologico della 'rottura dei livelli' (v.sopra), non stupisce che la parte del palo che viene interrata sia detta essere (TS 1,3,1,g) la sede dei Padri; sulla presenza di pali nelle tombe v.V. Moeller, Symbolik des Hinduismus und des Jainismus, Tafelband, Stuttgart 1974, p.10 e tav.7-8, che richiama RV 10,18,13 (tumulo della meta del I millennio a.C.): non so dire, tuttavia, se la funzione del pilastro sia quella religiosa sopra discussa.

tura animata) e spirituale (l'ispirazione poetica e religiosa); in quanto stimolatore di energie, può concederle sino al massimo grado (l'immortalità). Gli studiosi, peraltro, preoccupati come dicevo della rilevanza o meno del suo carattere solare, non sembrano essersi accorti che tali tratti sono comunque subordinati alla pertinenza 'varuniana' della figura divina nel suo insieme: ne ricordo qui alcuni tra quelli a mio avviso piu evidenti. Egli è un asura ( $\underline{\mathrm{RV}} 1,35,7$, ecc.), non di rado inserito tra gli āditya $(8,18,3$, ecc.), che opera attraverso rta e può legare con $i$ temibili lacci di Varuna $(10,85,24)$ : ha, come quest'ultimo, guardiani ineludibili $(6,71,3)$; e collegato alla Montagna primordiale $(7,37,8$, ecc.). Gli dei con i quali è più frequentemente citato sono Agni, Mitra, Sūrya (con i quali occasionalmente e identificato), Varuna e Bhaga (!). Come si vede, siamo di fronte a tutti quegli elementi splendidamente delineati dal Kuiper in " $\Pi J "$, sopra citato, ed attribuibili all'oscura e mistica dimora di Varuna; non ci stupiranno pertanto le frequenti connessioni di Savitar e la notte ed i suoi aspetti di sole diurno e notturno (Agni nella casa di V.), comunque ben distinguibili dall'immediata connotazione di Sūrya: in questa prospettiva la connessione sopra proposta con il pilastro cosmico è agevolmente accettabile. Tralascio ulteriori aspetti del dio, tutti peraltro riconducibili all'unità concettuale sin qui delineata (es.: in quanto stimolatore del movimento ordinato, egli regola le vie del movimento stesso [del sole, delle aurore, della notte, ecc.] con una regola che non puo essere infranta; questa conoscenza delle 'vie', atmosferiche [e da ciò deriva la sua caratterizzazione spaziale] e religiose [ le vie del/al sacrificio], lo fa avvicinare a Pūșan, talora con connotazioni funerarie; ecc. ). 
Lik Dyutāna Māruta it TS $1,3,1,1$ je že za antične pisce zelo dvomljiv; verjetno je Dyutāna zašlo v pasus vrātya zaradi patronimika Māruta. Verz iz TS. je bil pokvarjen že $v$ davnih časih; nitāná- "shoot of a plant which grows downwards" je iz nyagrōdha- "Ficus indica" in predstavlja pravzaprav axis mundi kozmično drevo, in to je bilo $\mathrm{v}$ Indiji mišljeno kot obrnjeno navzdol. Zato je Dy. M. nadomestiti z nitānás... mārutó; verz iz TS. je tedaj treba popraviti $v$ nitānás tvám mārutó "ti si dol obrnjeno marutsko deblo"... 